\title{
Polarizers tuned at key far-UV spectral lines for space instrumentation
}

\author{
Juan I. Larruquert ${ }^{1 *}$, A. Marco Malvezzi ${ }^{2,3}$, Luis Rodríguez-de Marcos ${ }^{1}$, Angelo Giglia ${ }^{2}$, \\ Nuria Gutiérrez-Luna ${ }^{1}$, Lucía Espinosa-Yáñez ${ }^{1}$, Carlos Honrado-Benítez ${ }^{1}$, José A. \\ Aznárez ${ }^{1}$, Giuseppe Massone ${ }^{3}$, Gerardo Capobianco ${ }^{3}$, Silvano Fineschi ${ }^{3}$, Stefano \\ Nannarone $^{2}$ \\ ${ }^{1}$ GOLD-IO-CSIC Instituto de Óptica-Consejo Superior de Investigaciones Científicas, \\ Madrid, Spain \\ ${ }^{2}$ Istituto Officina dei Materiali -Consiglio Nazionale delle Ricerche, Trieste, Italy \\ ${ }^{3} \mathrm{INAF}$ - Osservatorio Astrofisico di Torino, Torino, Italy
}

\begin{abstract}
Polarimetry is a valuable technique to help us understand the role played by the magnetic field of the coronal plasma in the energy transfer processes from the inner parts of the Sun to the outer space. Polarimetry in the far ultraviolet (FUV: 100-200 nm), which must be performed from space due to absorption in terrestrial atmosphere, supplies fundamental data of processes that are governed by the Doppler and Hanle effects on resonantly scattered line-emission. To observe these processes there are various key spectral lines in the FUV, from which H I Lyman $\alpha(121.6 \mathrm{~nm})$ is the strongest one. Hence some solar physics missions that have been proposed or are under development plan to perform polarimetry at $121.6 \mathrm{~nm}$, like the suborbital missions CLASP I (2015) and CLASP II (2018), and the proposed solar missions SolmeX and COMPASS and stellar mission Arago. Therefore, the development of efficient FUV linear polarizers may benefit these and other possible future missions. C IV $(155 \mathrm{~nm})$ and $\mathrm{Mg}$ II $(280 \mathrm{~nm})$ are other spectral lines relevant for studies of solar and stellar magnetized atmospheres.

High performance polarizers can be obtained with optimized coatings. Interference coatings can tune polarizers at the spectral line(s) of interest for solar and stellar physics. Polarizing beamsplitters consist in polarizers that separate one polarization component by reflection and the other by transmission, which enables observing the two polarization components simultaneously with a single polarizer. They involve the benefit of a higher efficiency in collection of polarization data due to the use of a single polarizer for the two polarization components and they may also facilitate a simplified design for a space polarimeter. We present results on polarizing beamsplitters tuned either at $121.6 \mathrm{~nm}$ or at the pair of 155 and $280 \mathrm{~nm}$ spectral lines.
\end{abstract}

\section{INTRODUCTION}

Measuring the magnetic field in the chromosphere, solar corona, and the transition region is a key objective for the solar physics community since the magnetic field controls the structure and dynamics of the solar atmosphere. Polarimetry in selected UV and far UV (FUV, wavelength shorter than $200 \mathrm{~nm}$ ) spectral lines is the best candidate technique to map the magnetic field in the solar atmosphere since most of the spectral lines that are sensitive to the magnetic field lie in the UV-FUV. UV and FUV polarimetry can provide measurements of the magnetic field through the evaluation of the Zeeman and Hanle effects. Other than solar physics, the magnetic field plays a fundamental role also in various fields of Astrophysics. One difficulty for polarization measurements is that a UV polarimeter must operate above the terrestrial atmosphere to avoid radiation absorption. After some pioneering solar missions devoted to FUV polarimetry from space, next developing or proposed solar physics missions, such as CLASP 1 and

\footnotetext{
*j.larruquert@csic.es; phone: 34- 915618806 ext 367; fax: 34-914117651
} 
2 [1,2], SolmeX [3], and SCORE [4], plan to perform improved polarimetry in the UV and FUV. Key spectral lines for polarimetry of the solar atmosphere are H Lyman $\alpha(121.6 \mathrm{~nm})$ and $\beta(102.6 \mathrm{~nm})$, O VI doublet (103.2 and $103.8 \mathrm{~nm})$, C IV (155 nm), and, in the UV, Mg II k line (280 nm).

Polarizers are essential optical elements for polarimetry and for other techniques like ellipsometry. The absorption of FUV radiation by most materials complicates the development of optical instruments to wavelengths as short as $121.6 \mathrm{~nm}$ or even more to $102.6 \mathrm{~nm}$. Bulk transmission elements operating by birefringence, like Rochon or Wollaston prisms have little or no capability to operate at wavelengths as short as $121.6 \mathrm{~nm}$. $\mathrm{MgF}_{2}$ Wollaston prisms have been proposed for astronomical polarimetry in the far UV [5]. This type of polarizing beam-splitter, however, has relatively small deviation angles $\left(\sim 20^{\circ}\right)$ requiring relatively large optical paths, what reduces the polarizer throughput. Wire-grid polarizers, which have been successfully extended to wavelengths in the deep UV [6], are not available in the FUV.

Polarizers based on coatings are a good choice when polarization is needed at a specific wavelength. From the pioneering designs of Kim et al. [7], coating polarizers with promising performance operating at Lyman $\alpha$ have been developed [8,9]. In addition to polarizers operating by reflectance, polarizers operating by transmittance have been also developed [9]; their smaller performance is compensated with the simpler geometry that involves no beam tilt. Transmittance polarizers can be also designed to additionally filter long wavelengths [9].

The present research goes one more step towards polarizers that satisfy new requirements: polarizing beamsplitters that simultaneously select one polarization component by reflection and the perpendicular component by transmission. The objective is to be able to separate the two polarization components with a single device, instead of the conventional use of two crossed polarizers. Such polarizers may be an important simplification for future space-operating polarimeters to be developed by the solar-physics community.

We present experimental data on polarizing beamsplitters for Lyman $\alpha$ with good polarization performance in terms of modulation factor and figure of merit. The same concept has been applied to polarizers tuned at two of the aforementioned spectral lines for solar atmosphere polarimetry: 155 and $280 \mathrm{~nm}$.

\section{EXPERIMENTAL TECHNIQUES}

Polarizers were designed and prepared at Grupo de Óptica de Láminas Delgadas (GOLD). Polarizers tuned at $121.6 \mathrm{~nm}$ were prepared with a multilayer alternating $\mathrm{Al}$ and $\mathrm{MgF}_{2}$ films; $\mathrm{Al}$ and $\mathrm{MgF}_{2}$ are the pair of standard materials for coatings in the FUV. Polarizers for 155 and $280 \mathrm{~nm}$ were prepared with a multilayer alternating $\mathrm{Al}$ and $\mathrm{AlF}_{3}$ films. Polarizers for a wavelength as long as $280 \mathrm{~nm}$ involve multilayer coatings with much thicker layers compared to $121.6 \mathrm{~nm}$; a trend observed on $\mathrm{MgF}_{2}$ films to increase porosity with thickness took us to switch this material for $\mathrm{AlF}_{3}$ in the multilayers for $280 \mathrm{~nm}$; $\mathrm{AlF}_{3}$ layers are expected to grow with a higher packing density compared with $\mathrm{MgF}_{2}$ ones. Besides, $\mathrm{AlF}_{3}$ displays similar optical properties to $\mathrm{MgF}_{2}$ for coatings tuned at much longer wavelengths.

$\mathrm{MgF}_{2}$ or quartz crystals were used as substrates for polarizers tuned at $121.6 \mathrm{~nm}$ or at 155 and $280 \mathrm{~nm}$, respectively. They were cut so that the optic axis of the crystal is perpendicular to the flat surfaces. Al, $\mathrm{MgF}_{2}$, and $\mathrm{AlF}_{3}$ films were deposited by evaporation on substrates at room temperature. $\mathrm{MgF}_{2}$ and $\mathrm{AlF}_{3}$ were evaporated using $\mathrm{W}$ boats and $\mathrm{Al}$ was evaporated using $\mathrm{W}$ filaments. $99.999 \%$ pure $\mathrm{Al}, 99.99 \%$ pure $\mathrm{AlF}_{3}$, and VUV-grade $\mathrm{MgF}_{2}$ were used as evaporant materials. Polarizer coatings were prepared in a 75cm diameter, oil-free stainless-steel chamber pumped with a cryopump. The chamber is placed in an ISO6 clean room.

Coating polarizers were measured at the BEAR (Bending magnet for Emission, Absorption and Reflectivity) beamline at Elettra synchrotron (Trieste, Italy). BEAR equipment permits optical spectroscopic study of the interfaces of multilayer systems [10,11,12], of anisotropic systems [13], and it is prepared for the characterization of polarizers $[9,14]$.

Direct transmittance and specular reflectance measurements of polarizers in $s$ (TE) and $p$ (TM) polarization $\left(T_{s}\right.$ and $T_{p}$, and $R_{s}$ and $R_{p}$, respectively), were obtained between $3 \mathrm{eV}(412 \mathrm{~nm})$ and $10.7 \mathrm{eV}$ 
$(116 \mathrm{~nm})$ with linearly polarized light. The degree of linear polarization had been previously determined to be $\left(\left(\mathrm{I}_{\mathrm{s}}-\mathrm{I}_{\mathrm{p}}\right) /\left(\mathrm{I}_{\mathrm{s}}+\mathrm{I}_{\mathrm{p}}\right)=0.99\right.$. The accuracy of angles of incidence is estimated to be $\sim 0.1^{\circ}$ for both transmission and reflection measurements. A variable aperture slit (see BEAR web [15]) accepting light of the bending magnet emission in an angle range within $\pm 0.17 \mathrm{mrad}$ with respect to the synchrotron orbit plane was used to set the degree of polarization. The cross section of the monochromatic light spot at the focus position was set at $400 \mathrm{x} 400 \mu \mathrm{m}^{2}$ horizontal $\mathrm{x}$ vertical; divergence and energy band width were $\sim 20$ $\mathrm{mrad}$ and $\sim 60 \mathrm{meV}\left(\Delta E / E=10^{-2}\right)$, respectively. The incident flux was $\sim 10^{9}$ photons/s. Rejection of higher diffraction orders was made by means of an optical grade thick LiF crystal filter from Crystec. Two alternate methods were used to measure the light polarization at the sample site. In one case a single analyzer element - which consisted in a LiF crystal slab mounted at the Brewster angle of the energy range at issue- was rotated together with the light detector mounted on the specular direction as a rigid assembly around the impinging light axis (see for instance Ref. [16]). In the other case $R_{s}$ and $R_{p}$, were measured with the LiF slab mounted at the Brewster angle and the major and minor axes of polarization of the ellipses were retrieved. The detector and sample assembly rotated rigidly around the impinging light axis in order to obtain the two polarization incidence geometries without changing the relative sample-detector position. A silicon diode (IRD-AXUV100) was used as light detector. Measurements were taken in DC current by a picoammeter. The impinging flux was monitored by measuring the refocusing-mirror drain current with a picoammeter.

Transmission and reflectivity data were obtained by averaging at each energy a set of light and dark (eventually subtracted) measurements. The overall accuracy of this method resulted in a statistical error $\sigma$ of the single measurement $\sim 10^{-13} \mathrm{~A}$ resulting in a lowest measurable flux (both in transmission and reflection) $\sim 10^{6}$ photons/s with a detection dynamics of three orders of magnitude. For both transmittance and reflectance measurements, the beam impinged on the sample from the coating side.

\section{RESULTS AND DISCUSSION}

The use of polarizing beamsplitters is a benefit for solar-atmosphere polarimeters since they make it possible to observe a certain field simultaneously in $\mathrm{s}$ and in $\mathrm{p}$ polarization using a simple geometry with reduced radiation loss due to the use of only one element. In some FUV polarimeters, such as CLASP, simultaneous observation of the two polarization components is performed using two symmetric diffraction orders of a grating as a beamsplitter; with this configuration, the overall efficiency is expected to be reduced due to light diffracted in other undesired orders.

\subsection{Polarizers at $121.6 \mathrm{~nm}$}

Our previous work addressed coating polarizers for $121.6 \mathrm{~nm}[9,17]$. Research on polarizers started with elements operating by reflectance and continued with novel polarizers operating by transmittance. Transmittance polarizers were seen to provide an extra benefit: they can be simultaneously used as transmittance filters, so that two functions are obtained with a single element and with no tilt of the radiation beam.

The total radiation balance after interaction with an optical element is distributed among reflection, transmission, and absorption. The degrees of freedom present in the design of a multilayer make it possible to optimize it for a given far-from-the-normal incidence angle so that one polarization component can be mostly reflected and the other component reasonably transmitted, the remaining energy being absorbed.

In our previous research [9], the target in polarizer design was to obtain a strong cancellation of one polarization component with an acceptable performance in the other component. This condition can be somewhat relaxed in its application for a space polarimeter so that the polarizer only requires that the performance on one component be significantly larger than on the other. To quantify this, the modulation factor is a useful evaluation parameter [18]:

$$
\mu_{T}=\frac{T_{p}-T_{s}}{T_{p}+T_{s}}
$$


where $T_{p}$ and $T_{s}$ represent the transmittance of the polarizer for the $p(T M)$ and $s(T E)$ polarization components, respectively. For a reflective polarizer, an equivalent parameter $\mu_{R}$ is defined by replacing $T_{p}$ and $T_{s}$ with $R_{s}$ and $R_{p}$, respectively. The top possible value for $\mu$ is 1 . The goal of reaching a high modulation factor must be compatible with an optimum use of the incoming energy. For this, the figure of merit $\kappa$ is a useful parameter:

$$
\kappa_{T}=\mu_{T} \sqrt{T_{\text {aver }}}
$$

where $T_{\text {aver }}$ is the transmittance averaged over the two polarizations: $T_{\text {aver }}=\left(T_{s}+T_{p}\right) / 2$, which will be referred to as throughput. $\kappa$ provides information on the trade-off between polarization efficiency and throughput. As for the modulation factor, an equivalent evaluation parameter $\kappa_{R}$ is defined for the reflective polarizer by replacing average transmittance with average reflectance. The top possible value for $\kappa$ is $2^{-1 / 2}=0.707$.
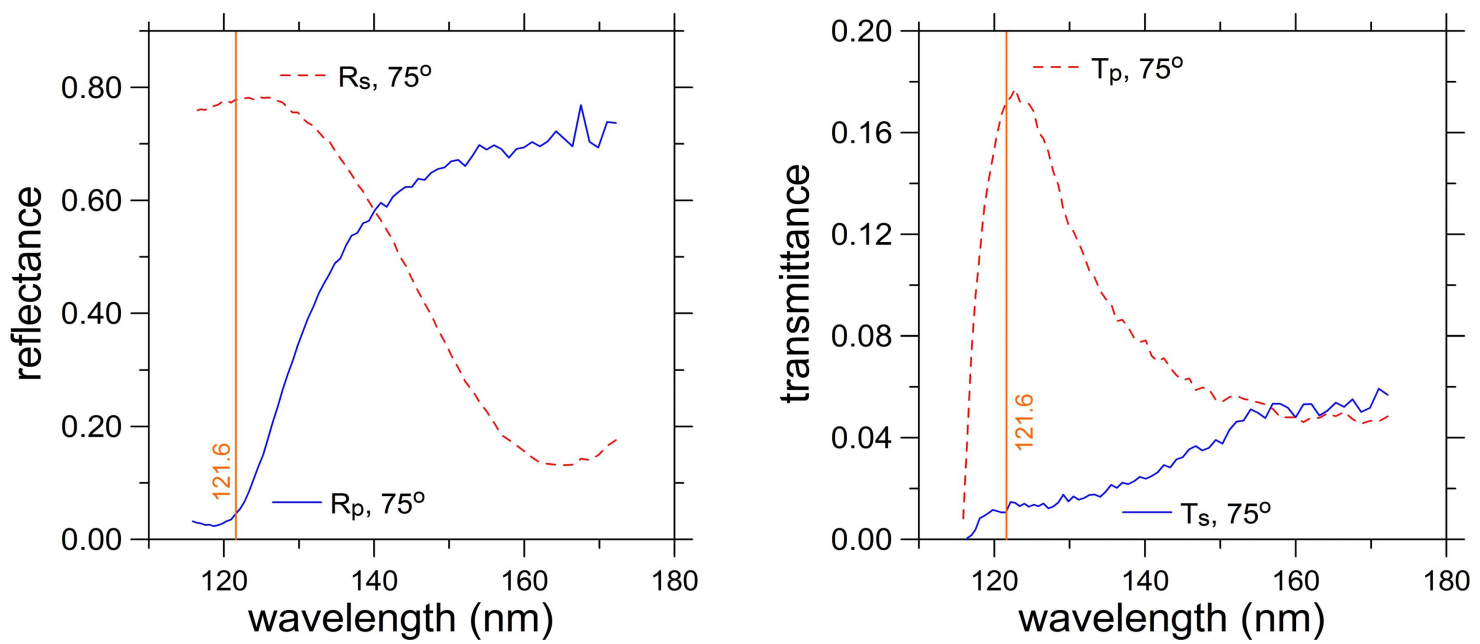

Fig. 1. s and p components of reflectance (left) and transmittance (right) at $75^{\circ}$ of a polarizing beamsplitter optimized in $\kappa$ at $121.6 \mathrm{~nm}$
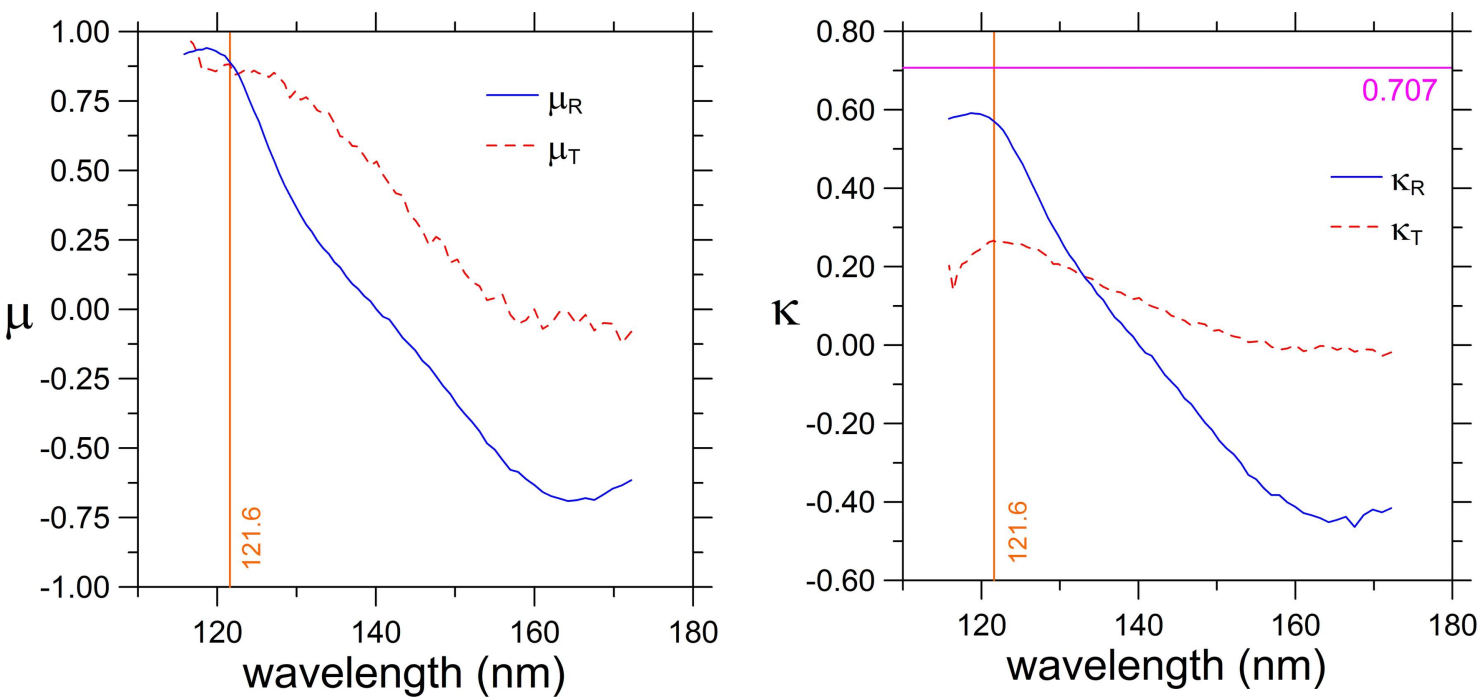

Fig. 2. $\mu$ (left) and $\kappa$ (right) at $75^{\circ}$ of a polarizing beamsplitter optimized in $\kappa$ at $121.6 \mathrm{~nm}$. The top $\kappa$ value of 0.707 is plotted for reference 
In the design of a reflective and transmissive polarizer we optimized the pair $\kappa_{R}$ and $\kappa_{T}$ in order to maximize throughput. Fig. 1 plots reflectance and transmittance measured for a reflective and transmissive polarizer optimized at $121.6 \mathrm{~nm}$.

Table 1. Modulation factor $\mu$ and figure of merit $\kappa$ at $121.6 \mathrm{~nm}$ both in reflectance and in transmittance of the polarizer plotted in Figs. 1 and 2

\begin{tabular}{|c|c|c|c|c|}
\hline Angle & $\boldsymbol{\mu}_{\mathrm{T}}$ & $\boldsymbol{\kappa}_{\mathbf{T}}$ & $\boldsymbol{\mu}_{\mathbf{R}}$ & $\boldsymbol{\kappa}_{\mathbf{R}}$ \\
\hline $75^{\circ}$ & 0.883 & 0.267 & 0.890 & 0.571 \\
\hline
\end{tabular}

Figure 2 displays evaluation parameters $\mu$ and $\kappa$ calculated for data plotted in Fig. 1. Table 1 summarizes the evaluation parameters at $121.6 \mathrm{~nm} . \mu$ has similar values for reflectance and transmittance, which results in that the capacity to discriminate one polarization or the other is similar by reflection and by transmission. On the other hand, $\kappa$ is considerably larger for reflectance than for transmittance, which expresses that the throughput is much larger in the reflectance section. The reason for this is that polarization by transmittance involves absorption mostly in the Al films and in the substrate. This asymmetry is intrinsic to the present $\mathrm{Al} /$ fluoride multilayers; it might be reduced by optimizing the multilayer at a less grazing incidence, although the asymmetry correction would result in a reduction of the performance in reflectance more than in an increase of the performance in transmittance. One could take advantage of this asymmetry in a situation in which one polarization component is smaller than the other and is therefore analyzed by reflectance.

The present coating polarizers were deposited on $\mathrm{MgF}_{2}$ substrates. $\mathrm{MgF}_{2}$ crystals are birefringent, which results in a modification of the wave polarization properties upon transmission through the crystal. This could be a problem if the polarized light that is transmitted through the coating were required to keep its polarization properties through the optical instrument; this problem could be solved by using a $\mathrm{MgF}_{2}$ substrate cut with the optic axis in the propagation direction within the crystal or by using LiF, an isotropic material, as a substrate. But if polarized light at the exit of the polarizer is immediately detected by means of a detector that is assumed to be polarization independent, birefringence-induced depolarization does not affect the measurements of the degree of linear polarization performed with the polarimeter.

\subsection{Polarizers at 155 and $280 \mathrm{~nm}$}

The present approach of multilayer polarizers reflecting one component and transmitting the other can be taken to simultaneously polarize C IV $(155 \mathrm{~nm})$ and Mg II $(280 \mathrm{~nm})$ spectral lines. Efficient reflective wire-grid polarizers operating at $280 \mathrm{~nm}$ are already available nowadays, but not at a wavelength as short as $155 \mathrm{~nm}$. In contrast, a multilayer coating is a promising polarizer candidate to cover the two wavelengths.

For these polarizers, the $\mathrm{Al} / \mathrm{AlF}_{3}$ multilayers were designed with simultaneous requirements to maximize $\kappa_{\mathrm{R}}$ and $\kappa_{\mathrm{T}}$ both at 155 and at $280 \mathrm{~nm}$. Figs. 3 and 4 display reflectance and transmittance along with their evaluation parameters, respectively, measured for one such polarizer. Table 2 summarizes the evaluation parameters of this polarizing beamsplitter at 155 and $280 \mathrm{~nm}$. 

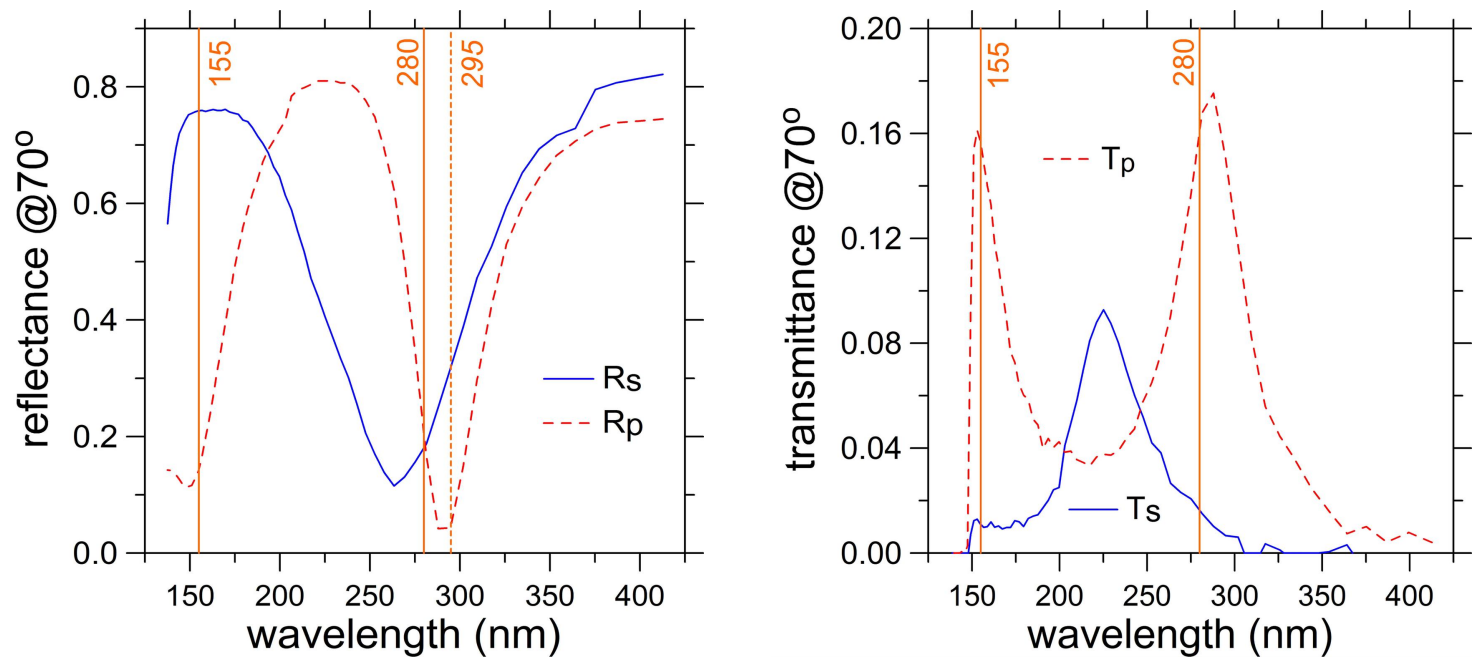

Fig. 3. $\mathrm{s}$ and p components of reflectance (left) and transmittance (right) at $70^{\circ}$ of a polarizing beamsplitter optimized both at 155 and $280 \mathrm{~nm}$
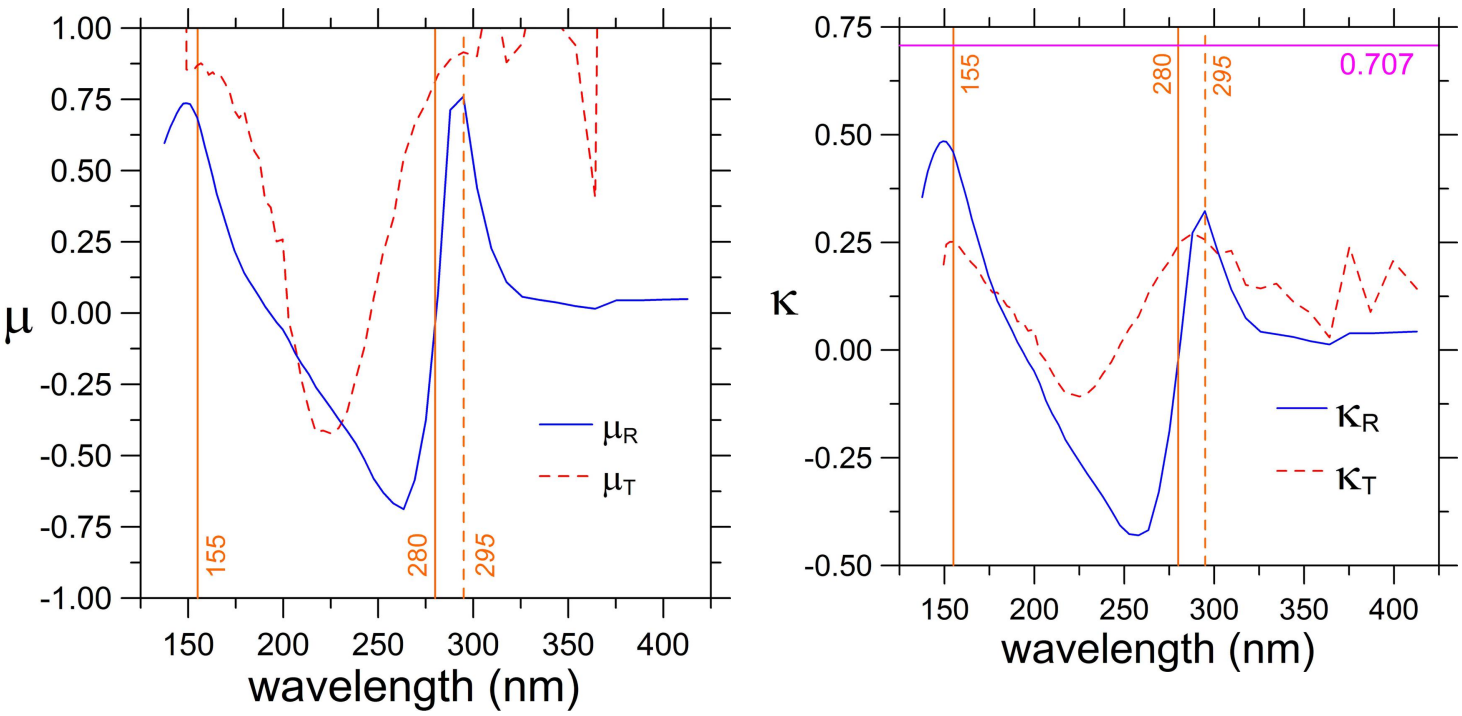

Fig. 4. $\mu$ (left) and $\kappa$ (right) at $70^{\circ}$ of a polarizing beamsplitter optimized in $\kappa$ at 155 and $280 \mathrm{~nm}$. The top $\kappa$ value of 0.707 is plotted for reference

The evaluation parameters are rather satisfactory, except for reflectance at $280 \mathrm{~nm}$. The feature designed at $280 \mathrm{~nm}$ seems to have shifted to the close wavelength of $295 \mathrm{~nm}$. This shift can be attributed to inaccuracies in the optical constants of $\mathrm{AlF}_{3}$. New polarizers will be prepared to try to move this highpolarization feature to $280 \mathrm{~nm}$ without destroying the good performance at $155 \mathrm{~nm}$.

Table 2. Modulation factor $\mu$ and figure of merit $\kappa$ at 155 and $280 \mathrm{~nm}$ both in reflectance and in transmittance of the polarizer plotted in Figs. 3 and $4^{\mathrm{a}}$

\begin{tabular}{|c|c|c|c|c|c|c|c|c|c|c|}
\hline Angle & $\mu_{\mathrm{T} 155}$ & $\kappa_{\mathrm{T} 155}$ & $\mu_{\mathrm{R} 155}$ & $\kappa_{\mathrm{R} 155}$ & $\mu_{\mathrm{T} 280}$ & $\boldsymbol{\kappa}_{\mathrm{T} 280}$ & $\mu_{\mathrm{R} 280}$ & $\kappa_{\mathrm{R} 280}$ & $\mu_{R 295}$ & $\boldsymbol{\kappa}_{R 225}$ \\
\hline $70^{\circ}$ & 0.869 & 0.251 & 0.680 & 0.457 & 0.834 & 0.244 & -0.02 & 0.00 & 0.750 & 0.318 \\
\hline
\end{tabular}

${ }^{\mathrm{a}}$ : reflectance $\mu$ and $\kappa$ are also given at $295 \mathrm{~nm}$

\section{SUMMARY AND CONCLUSIONS}

Polarizing beamsplitters have been developed for two targets with application in solar atmosphere: 121.6 $\mathrm{nm}(\mathrm{H}$ Lyman $\alpha)$ and the pair 155 (C IV) and $280 \mathrm{~nm}$ (Mg II). They consist in coating polarizers based in 
a multilayer of $\mathrm{Al} / \mathrm{MgF}_{2}(121.6 \mathrm{~nm})$ or $\mathrm{Al} / \mathrm{AlF}_{3}(155$ and $280 \mathrm{~nm})$. Multilayers were designed to optimize the figure of merit as the evaluation parameter. The performance of the 121.6-nm polarizer was found satisfactory. The performance of the $155-\& 280-\mathrm{nm}$ polarizer was found mostly satisfactory, except for a shift in performance for the reflectance at $280 \mathrm{~nm}$. A modified design is planned to move the shifted feature to the correct wavelength while keeping the other parameters satisfactory.

The developed polarizers are able to separate the two polarization components with a single device, which results in an increased performance over the use of two crossed polarizers after a beamsplitter. Additionally, polarizing beamsplitters may be an important simplification for future space-operating polarimeters to be developed by the solar-physics community.

\section{ACKNOWLEDGMENTS}

We acknowledge support by the European Community-Research Infrastructure Action under the FP6 'Structuring the European Research Area' Programme (through the Integrated Infrastructure Initiative 'Integrating Activity on Synchrotron and Free Electron Laser Science'); measurements were performed under ELETTRA proposal numbers 20115134, 20120059, 20125119, 20130201, 20135237, 20140171, 20150344, and 2015497. This work was also supported by the National Programme for Research, Subdirección General de Proyectos de Investigación, Ministerio de Ciencia e Innovación, project numbers AYA2013-42590-P and ESP2016-76591-P. We also acknowledge support by the Italian Ministry of University and Research (MIUR) under the Programmi di Ricerca Scientifica di Rilevante Interesse Nazionale-Bando 2012.

\section{REFERENCES}

[1] Narukage N., McKenzie D. E., Ishikawa R., Trujillo-Bueno De Pontieu J., B., Kubo M., Ishikawa S., Kano R., Suematsu Y., Yoshida M., Rachmeler L. A., Kobayashi K., Cirtain J. W., Winebarger A. R., Ramos A. A., del Pino Aleman T., Stepan J., Belluzzi L., Larruquert J. I., Auchère F., Leenaarts J., and Carlsson M. J. L., "Chromospheric LAyer SpectroPolarimeter (CLASP2)," Proc. SPIE 9905, 990508 (2016).

[2] Kano R., Bando T., Narukage N., Ishikawa R., Tsuneta S., Katsukawa Y., Kubo M., Ishikawa S., Hara H., Shimizu T., Suematsu Y., Ichimoto K., Sakao T., Goto M., Kato Y., Imada S., Kobayashi K., Holloway T., Winebarger A., Cirtain J., De Pontieu B., Casini R., Trujillo Bueno J., Stepan J., Manso Sainz R., Belluzzi L., Ramos A. A., Auchére F., and Carlsson M., "Chromospheric Lyman-Alpha Spectro-Polarimeter (CLASP)," Proc. SPIE 8443, 84434F (2012).

[3] Peter H., Abbo L., Andretta V., Auchère F., Bemporad A., Berrilli F., Bommier V., Braukhane A., Casini R., Curdt W., Davila J., Dittus H., Fineschi S., Fludra A., Gandorfer A., Griffin D., Inhester B., Lagg A., Landi Degl'Innocenti E., Maiwald V., Manso Sainz R., Martínez Pillet V., Matthews S., Moses D., Parenti S., Pietarila A., Quantius D., Raouafi N.-E., Raymond J., Rochus P., Romberg O., Schlotterer M., Schühle U., Solanki S., Spadaro D., Teriaca L., Tomczyk S., Trujillo Bueno J., Vial J.-C., "Solar magnetism eXplorer (SolmeX)," Exp. Astron. 33 271-303 (2012).

[4] Fineschi S., Antonucci E., Romoli M., Gardiol D., Naletto G., Giordano S., Malvezzi M., Da Deppo V., Zangrilli L., Noci G.., "Ultraviolet and Visible-light Coronagraphic Imager (UVCI) for HERSCHEL," Proc. SPIE 4853, 162-171 (2003).

[5] Pertenais M., Neiner C., Parès L. P., Petit P., Snik F., van Harten G., “UVMag: Space UV and visible spectropolarimetry," Proc. SPIE 9144, 91443B (2014).

[6] Siefke T., Kroker S., Pfeiffer K., Puffky O., Dietrich K., Franta D., Ohlídal I., Szeghalmi A., Kley E.B., and Tünnermann A., "Materials Pushing the Application Limits of Wire Grid Polarizers further into the Deep Ultraviolet Spectral Range," Adv. Optical Mater. 4, 1780-1786 (2016).

[7] Kim J., Zukic M., and Torr D. G., "Multilayer thin film design as far ultraviolet polarizers," Proc. SPIE 1742, 413-22 (1992).

[8] Bridou F., Cuniot-Ponsard M., Desvignes J.-M., Gottwald A., Kroth U., Richter M., "Polarizing and non-polarizing mirrors for the hydrogen Lyman- $\alpha$ radiation at $121.6 \mathrm{~nm}$," Appl Phys A 102, 641-649 (2011). 
[9] Larruquert J. I., Malvezzi A. M., Giglia A., Aznárez J. A., Rodríguez-de Marcos L., Méndez J. A., Miotti P., Frassetto F., Massone G., Nannarone S., Crescenzio G., Capobianco G., Fineschi S., "Reflective and transmissive coating polarizers in a spectral range centered at $121.6 \mathrm{~nm}$," J. Opt. 16, 125713 (2014).

[10] Mahne, N., Giglia A., Sponza L., Verna A., Nannarone S., "Soft-X study of buried interfaces in stratified media," Proc. SPIE 7995, 79951S (2010).

[11] Giglia A., Mahne N., Bianco A., Svetin, C., Nannarone S., "EUV soft X-ray characterization of a FEL multilayer optics damaged by multiple shot laser beam," Nucl. Instr. Meth. A 635, S30-S38 (2011).

[12] Jonnard P., Le Guen K., André J.-M., Delaunay R., Mahne N., Giglia A., Nannarone S., Vern, A., Wang Z.-S., Zhu J.-T., and Zhou S.-K., "Determination of the magnetization profile of $\mathrm{Co} / \mathrm{Mg}$ periodic multilayers by magneto-optic Kerr and X-ray magnetic resonant reflectivity,” J. Phys.: Conf. Ser. 417, 012025 (2013).

[13] Pasquali L., Mukherjee S., Terzi F., Giglia A., Mahne N., Koshmak K., Esaulov V., Toccafondi C., Canepa M., Nannarone S., "Structural and electronic properties of anisotropic ultrathin organic films from dichroic resonant soft x-ray reflectivity,” Phys. Rev. B 89, 045401 (2014).

[14] Tan M. Y., Zhu J. T., Chen L. Y., Wang Z. S., Le Guen K., Jonnard P., Gigli, A., Mahne N., Nannarone S., "Molybdenum-silicon aperiodic multilayer broadband polarizer for 13-30 nm wavelength range," Nucl. Instr. Meth. A 654, 588-591 (2011).

[15] http://www.elettra.trieste.it/it/lightsources/elettra/elettra-beamlines/bear/bear.html

[16] Schäfers F., Mertins H.-C., Gaupp A., Gudat W., Mertin M., Packe I., Schmolla F., Di Fonzo S., Soullié G., Jark W., Walker R., Le Cann X., Nyholm R., and Eriksson M., "Soft-X-ray polarimeter with multilayer optics: complete analysis of the polarization state of light," Appl. Opt. 38, 4074-4088 (1999).

[17] Larruquert J. I., Malvezzi A. M., Giglia A., Aznárez J. A., Rodríguez-de Marcos L., Méndez J. A., Miotti P., Frassetto F., Massone G., Capobianco G., Fineschi S., Nannarone S., "Polarizers for a spectral range centered at $121.6 \mathrm{~nm}$ operating by reflectance or by transmittance," Proc. SPIE 9510, 951008 (2015).

[18] Fineschi S., Gardner D., Kohl J. L., Romoli M., Pace E., Corti G., Noci G., "Polarimetry of the UV Solar Corona with ASCE," Proc. SPIE 3764, 147-160 (1999). 